\title{
The Relationship Between Sleep Disturbance and Functional Status in Mild Stroke Patients
}

\author{
Jinil Kim, MD ${ }^{1}$, Yuntae Kim, MD ${ }^{1}$, Kwang Ik Yang, MD $^{2}$, Doh-eui Kim, MD², Soo A Kim, MD ${ }^{1}$ \\ ${ }^{1}$ Department of Physical Medicine and Rehabilitation, Soonchunhyang University Cheonan Hospital, Cheonan; \\ ${ }^{2}$ Sleep Disorders Center, Department of Neurology, Soonchunhyang University Cheonan Hospital, Cheonan, Korea
}

Objective To investigate the sleep state of mild stroke patients and relationship between sleep disturbance and functional status.

Methods A total of 80 acute stroke patients were enrolled in this study. The criteria for inclusion in the study was as following: 1) first stroke, 2) cognitive function preserved enough to perform the test (Mini Mental State Examination $\geq 24$ ), 3) good functional levels (Modified Rankin Scale $\leq 3$ ), 4) upper extremity motor function preserved enough to perform occupational tests (hand strength test, Purdue pegboard test, 9-hole peg test, and Medical Research Council score $\geq 3$ ), and 5) less than 2 weeks between the stroke and the assessment. Quality of sleep was assessed by using Pittsburg Sleep Quality Index (PSQI), Epworth Sleepiness Scale (ESS), Insomnia Severity Index (ISI), and Stanford Sleepiness Scale (SSS). Activities of daily living was assessed by using the Modified Barthel Index (MBI) and depressed mood was assessed by using the Beck Depression Inventory (BDI). Gross and fine motor function of the upper extremity was assessed by using hand strength test (Jamar dynamometer), Purdue pegboard test, and the 9-hole peg test.

Results The results of the occupational assessment were fine in the good sleepers. The PSQI, ESS, and ISI were correlated with some of the assessment tools (BDI, MBI, Purdue pegboard, 9-hole peg, and hand strength).

Conclusion In conclusion, this study emphasizes that sleep disturbance can affect the functional status in mild acute stroke patients. Therefore, clinicians must consider sleep status in stroke patients and need to work to control it.

Keywords Stroke, Sleep, Hand function

Received August 20, 2014; Accepted December 12, 2014

Corresponding author: Soo A Kim

Department of Physical Medicine and Rehabilitation, Soonchunhyang University Cheonan Hospital, 31 Suncheonhyang 6-gil, Dongnam-gu, Cheonan 330-930, Korea

Tel: +82-41-570-2220, Fax: +82-41-576-3464, E-mail: sooapmr@schmc.ac.kr

(c) This is an open-access article distributed under the terms of the Creative Commons Attribution Non-Commercial License (http://creativecommons. org/licenses/by-nc/4.0) which permits unrestricted noncommercial use, distribution, and reproduction in any medium, provided the original work is properly cited.

Copyright @ 2015 by Korean Academy of Rehabilitation Medicine

\section{INTRODUCTION}

Stroke is a leading cause of disability in adults and accounts for a large percentage of disease, worldwide [1]. Mild stroke and transient ischemic attack now account for $82 \%$ of all cases of cerebrovascular disease and $60 \%$ of all cases of stroke [2]. While mild stroke patients can suffer from prolonged disability and discomfort, they are often discharged with little or no rehabilitation services that address their return to work [3]. As a result, when 
they return to real life, diminished quality of life and limitations in higher levels of physical functioning often persist. Difficulties with executive function, fatigue, sleep disturbance, and psychological symptoms (e.g., depression and anxiety) also impact their ability to return to society or home; a condition referred to as hidden dysfunction [4-8].

Survivors of stroke regard upper extremity function to be important for increasing their quality of life [9] and recovery of arm function seems to be largely independent of the overall severity of the stroke [10-12]. Psychological factors such as depression and sleep disturbance are associated with a poorer functional prognosis and poststroke fatigue [13-15].

Sleep disturbance occurs, or worsens, after a stroke and occurs in various forms such as sleep apnea, insomnia, and daytime sleepiness and has been known to disturb the life of rehabilitation stroke patients [16-19]. The aforementioned hidden dysfunction is minimized by effective rehabilitation treatment but if patients are affected by sleep disturbances, their rehabilitation treatment is not effective and their functional recovery is delayed [2023]. Mild stroke patients have more potential to recover their pre-stroke functional, and that is why it is important to control sleep disturbances. In previous studies, functional status of patients was measured with the Modified Barthel Index (MBI) or the Functional Independence Measurement (FIM). However, we also investigated upper extremity's functional status using further evaluation tool. Herein this study we aimed to investigate the sleep state of mild stroke patients and determine the relationship between sleep disturbance and functional status.

\section{MATERIALS AND METHODS}

\section{Participants}

This study was a prospective study. A total of 80 patients were enrolled in this study and were recruited from Soonchunhyang University Hospital between October 2013 and June 2014 (Fig. 1). The inclusion criteria were as following: 1) it was the patients' first stroke; 2 ) the Mini Mental State Examination [MMSE] score $\geq 24 ; 3$ ) the Modified Rankin Scale (MRS) score $\leq 3 ; 4$ ) upper extremity motor function preserved enough to perform occupational tests (hand strength test, Purdue pegboard test, 9-hole peg test, and a Medical Research Council [MRC] score $\geq 3$ ); and 5) the stroke occurred less than 2 weeks

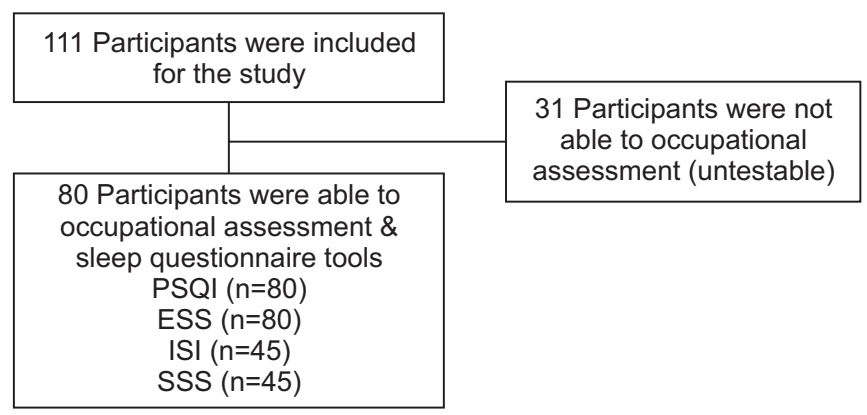

Fig. 1. This study was a prospective study. PSQI, Pittsburg Sleep Quality Index; ESS, Epworth Sleepiness Scale; ISI, Insomnia Severity Index; SSS, Stanford Sleepiness Scale.

from the start of the study. Patients were excluded from the study if they met any of the following criteria: 1) had a history of cerebrovascular accidents; 2 ) had a severe cognitive impairment, aphasia, or dementia; 3 ) had a psychiatric disorder such as schizophrenia, behavioral, or affective disorder; 4) had previously been treated for sleep problems; 5) were taking, or had taken, sleeping pills; and 6) had a history of depression.

This study was approved by the Institutional Review Board of Soonchunhyang University Hospital and informed consent was obtained from all patients.

\section{Assessment of sleep}

The patient's sleep states were assessed by using four sleep questionnaires: 1) the Pittsburgh Sleep Quality In$\operatorname{dex}(\mathrm{PSQI}), 2)$ the Epworth Sleepiness Scale (ESS), 3) the Insomnia Severity Index (ISI), and 4) the Stanford Sleepiness Scale (SSS). The PSQI is typically used for evaluating quantitative and subjective aspects of sleep, such as depth and comfort, which are both main considerations in the current study [24]. A PSQI score between 0 and 5 indicated that the patient was a good sleeper while a score greater than 5 was indicative of poor sleep $[24,25]$. The ESS is used to measure a patients average sleep propensity across different situations in daily life [26]. For the ESS, a score from 0-8 indicates good sleep and above 8 indicates poor sleep $[26,27]$. The ISI targets the subjective symptoms and consequences of insomnia as well as the degree of concerns or distress caused by those difficulties [28]. An ISI between 0 and 7 was representative of a good sleeper and a score greater than 7 was indicative of poor sleep [28]. The SSS is frequently used to assess subjective perceptions of daytime sleepiness [29]. The SSS is scored from 1-7 with a higher score related to a poorer sleep 
state [29]. All questionnaires were performed within 2 weeks of the stroke. If the patient was unable to complete the questionnaire on their own, a caregiver or family member assisted them.

\section{Functional impairments}

Activities of daily living were assessed by using the MBI $[30,31]$ while cognitive function was assessed by using the MMSE. Depression was assessed by using the Beck Depression Inventory (BDI), an easy to implement questionnaire and a score that exceeds 15 points indicates that the patient was depressed [32].

Upper extremity strength and function were assessed by using the hand strength test (Jamar dynamometer), Purdue pegboard test, and the 9-hole peg test. All tests were performed by the affected and unaffected sides and both the dominant and non-dominant hands were compared. The hand strength test (Jamar dynamometer) was made up of 4 components grip, tip, lateral, and jaw [3335]. The Purdue pegboard test was measured by calculating the number of pins the patient was able to place in the holes in 30 seconds $[36,37]$ while the 9-hole peg test measured the time (seconds) it took to put all of the pins in the holes [38]. All tests were performed by an occupational therapist while another occupational therapist was responsible for supervising and recording the data.

\section{Statistical analysis}

All statistical analyses were performed with SPSS statistical software ver. 17.0 (SPSS Inc., Chicago, IL, USA) and the independent t-test was used to compare the data obtained from good and poor sleepers. Spearman correlation analysis was used to assess the relationship between PSQI, ESS, ISI, and SSS and the independent variablesBDI, MBI, hand strength test (Jamar dynamometer), the Purdue pegboard test, and the 9-hole peg test. A p-value below 0.05 was considered statistically significant.

\section{RESULTS}

\section{Demographic and stroke characteristics}

Characteristics of the $\mathbf{8 0}$ patients included in study are summarized in Table 1. The mean patient age was $63.8 \pm 13.6$ years, and the mean interval between the onset of stroke and time of assessment was $6.8 \pm 6.0$ days. The

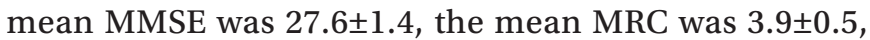

and the mean MRS was $2.2 \pm 0.8$. Seventy-six patients had infarcts and 4 had experienced hemorrhages. The lesions were located in the cortical and subcortical regions in 23 and 57 patients, respectively. Three patients were left handed and 77 were right handed.

\section{Sleep and functional impairment}

The average MBI score was $63.6 \pm 21.4$. The average Purdue peg test was $5.9 \pm 3.5$ on the affected side and 9.6 \pm 3.0 on the unaffected side. The average 9-hole peg test was $58.6 \pm 55.2$ on the affected side and $30.0 \pm 9.2$ on the unaffected side. The average grip hand strength was $21.5 \pm 12.1$ on the affected side and $27.6 \pm 12.1$ on the unaffected side. The average tip hand strength was $2.1 \pm 1.5$ on affected

Table 1. Demographic and stroke-related characteristics $(\mathrm{n}=80)$

\begin{tabular}{|lc|}
\hline Characteristic & Value \\
\hline Gender & \\
\hline Male & 54 \\
\hline Female & $63.8 \pm 13.6$ \\
\hline Age (yr) & \\
\hline Affected side & 35 \\
\hline Right & 45 \\
\hline Left & $2.2 \pm 0.8$ \\
\hline MRS & $27.6 \pm 1.4$ \\
\hline MMSE & $6.8 \pm 6.0$ \\
\hline Time since stroke (day) & $3.9 \pm 0.5$ \\
\hline MRC scale & \\
\hline Stroke type & 76 \\
\hline Ischemic & 4 \\
\hline Hemorrhagic & \\
\hline Lesion & 23 \\
\hline Cortical & 57 \\
\hline Subcortical & \\
\hline Dominant hand & 77 \\
\hline Right & 3 \\
\hline Left & 45.0 \\
\hline Comorbidity & 21.3 \\
\hline Hypertension (\%) & \\
\hline Diabetes mellitus (\%) & \\
\hline are presented as & \\
\hline
\end{tabular}

Values are presented as number or mean \pm standard deviation.

MRS, Modified Rankin Scale; MMSE, Mini-Mental State Examination; MRC, Medical Research Council. 
Table 2. The average scores of upper extremity hand functions listed by affected side in right handed patients $(\mathrm{n}=77)$

\begin{tabular}{|c|c|c|}
\hline & \multicolumn{2}{|c|}{ Affected side } \\
\hline & Right $(n=33)$ & Left $(n=44)$ \\
\hline \multicolumn{3}{|c|}{ Purdue peg } \\
\hline DH & $5.9 \pm 2.7^{*}$ & $10.1 \pm 3.3$ \\
\hline $\mathrm{NH}$ & $8.7 \pm 2.5$ & $5.6 \pm 3.8^{*}$ \\
\hline \multicolumn{3}{|c|}{ 9-Hole peg (s) } \\
\hline DH & $56.3 \pm 64.6^{*}$ & $28.7 \pm 9.2$ \\
\hline $\mathrm{NH}$ & $32.4 \pm 8.8$ & $62.7 \pm 49.0^{*}$ \\
\hline \multicolumn{3}{|c|}{ Strength (grip) } \\
\hline DH & $22.7 \pm 11.2$ & $27.4 \pm 11.7$ \\
\hline $\mathrm{NH}$ & $26.6 \pm 11.8$ & $19.6 \pm 11.7^{*}$ \\
\hline \multicolumn{3}{|c|}{ Strength (tip) } \\
\hline DH & $2.1 \pm 1.2^{*}$ & $3.5 \pm 2.4$ \\
\hline NH & $3.0 \pm 1.3$ & $2.0 \pm 1.8^{*}$ \\
\hline \multicolumn{3}{|c|}{ Strength (lateral) } \\
\hline DH & $4.9 \pm 1.9^{*}$ & $5.9 \pm 2.0$ \\
\hline $\mathrm{NH}$ & $5.9 \pm 1.8$ & $3.9 \pm 2.4^{*}$ \\
\hline \multicolumn{3}{|c|}{ Strength (jaw) } \\
\hline DH & $3.3 \pm 1.8^{*}$ & $4.7 \pm 1.8$ \\
\hline $\mathrm{NH}$ & $4.4 \pm 1.8$ & $2.7 \pm 2.1^{*}$ \\
\hline
\end{tabular}

Values are presented as mean \pm standard deviation.

$\mathrm{DH}$, dominant hand (right); $\mathrm{NH}$, non-dominant hand (left).

${ }^{*} \mathrm{p}<0.05$, derived from $\mathrm{t}$-test. side and $3.3 \pm 2.0$ unaffected side. The average lateral hand strength was $4.4 \pm 2.3$ on the affected side and 5.9 \pm 2.0 on the unaffected side. The average jaw hand strength was $3.0 \pm 2.0$ on the affected side and $4.6 \pm 1.8$ on the unaffected side.

Of the 77 patients who were right handed, 33 were affected in their right hand, while 44 were affected on their left side. The dominant and non-dominant hands had

Table 4. Correlation analysis (Spearman's rho) between post stroke impairments and the PSQI, ESS, ISI, and SSS scores

\begin{tabular}{lcccc}
\hline & $\begin{array}{c}\text { PSQI } \\
(\mathbf{n = 8 0})\end{array}$ & $\begin{array}{c}\text { ESS } \\
(\mathbf{n = 8 0})\end{array}$ & $\begin{array}{c}\text { ISI } \\
(\mathbf{n}=\mathbf{4 5})\end{array}$ & $\begin{array}{c}\text { SSS } \\
(\mathbf{n}=\mathbf{4 5})\end{array}$ \\
\hline BDI & $0.453^{* *}$ & $0.368^{* *}$ & $0.650^{* *}$ & $0.526^{* *}$ \\
MBI & -0.219 & -0.144 & $-0.305^{*}$ & $-0.318^{*}$ \\
Purdue peg & -0.060 & -0.137 & -0.227 & $-0.377^{*}$ \\
9-Hole peg (s) & 0.009 & 0.036 & -0.006 & 0.177 \\
Strength (grip) & $-0.232^{*}$ & -0.152 & $-0.396^{* *}$ & $-0.456^{* *}$ \\
Strength (tip) & -0.219 & -0.152 & $-0.430^{* *}$ & $-0.478^{* *}$ \\
Strength (lateral) & -0.219 & -0.137 & $-0.353^{*}$ & $-0.341^{*}$ \\
Strength (jaw) & -0.188 & -0.090 & $-0.370^{*}$ & $-0.463^{* *}$ \\
\hline
\end{tabular}

PSQI, Pittsburg Sleep Quality Index; ESS, Epworth Sleepiness Scale; ISI, Insomnia Severity Scale; SSS, Stanford Sleepiness Scale; BDI, Beck Depression Inventory; MBI, Modified Barthel Index.

${ }^{*} \mathrm{p}<0.05,{ }^{* *} \mathrm{p}<0.01$, Spearman's rho correlation coefficient.

Table 3. Post stroke impairments between patients with or without sleep disturbance

\begin{tabular}{|c|c|c|c|c|c|c|}
\hline & \multicolumn{2}{|c|}{ PSQI } & \multicolumn{2}{|c|}{ ESS } & \multicolumn{2}{|c|}{ ISI } \\
\hline & $\begin{array}{c}\text { Group } 1(n=23) \\
\text { Score } \leq 5\end{array}$ & $\begin{array}{c}\text { Group } 2(n=57) \\
\text { Score }>5\end{array}$ & $\begin{array}{c}\text { Group } 1(n=41) \\
\text { Score } \leq 8\end{array}$ & $\begin{array}{c}\text { Group } 2(n=39) \\
\text { Score }>8\end{array}$ & $\begin{array}{c}\text { Group } 1(\mathbf{n}=21) \\
\text { Score } \leq 7\end{array}$ & $\begin{array}{c}\text { Group } 2(n=24) \\
\text { Score }>7\end{array}$ \\
\hline BDI & $12.0 \pm 8.5^{\mathrm{a}, \mathrm{b})}$ & $17.7 \pm 8.3^{\mathrm{a}, \mathrm{b})}$ & $16.0 \pm 8.7$ & $16.1 \pm 8.7$ & $16.9 \pm 7.8$ & $14.8 \pm 8.6$ \\
\hline MBI & $65.0 \pm 17.3$ & $63.1 \pm 23.0$ & $62.3 \pm 20.4$ & $65.1 \pm 22.7$ & $64.6 \pm 20.1$ & $60.8 \pm 23.6$ \\
\hline Purdue peg & $6.0 \pm 3.3$ & $5.9 \pm 3.6$ & $6.3 \pm 3.7$ & $5.6 \pm 3.3$ & $5.6 \pm 3.5$ & $5.5 \pm 3.1$ \\
\hline 9-Hole peg (s) & $52.6 \pm 36.5$ & $61.0 \pm 61.3$ & $52.6 \pm 44.5$ & $64.9 \pm 64.6$ & $64.3 \pm 81.9$ & $64.8 \pm 42.0$ \\
\hline Strength (grip) & $24.2 \pm 11.4$ & $20.4 \pm 12.2$ & $23.2 \pm 12.4$ & $19.8 \pm 11.6$ & $23.2 \pm 9.9$ & $19.1 \pm 13.2$ \\
\hline Strength (tip) & $2.3 \pm 1.6$ & $2.0 \pm 1.5$ & $2.2 \pm 1.4$ & $1.9 \pm 1.7$ & $2.3 \pm 1.4$ & $1.7 \pm 1.8$ \\
\hline Strength (lateral) & $4.8 \pm 2.3$ & $4.2 \pm 2.3$ & $4.6 \pm 2.3$ & $4.2 \pm 2.3$ & $4.8 \pm 2.2$ & $3.8 \pm 2.2$ \\
\hline Strength (jaw) & $3.5 \pm 2.1$ & $2.8 \pm 1.9$ & $3.2 \pm 1.9$ & $2.8 \pm 2.1$ & $3.1 \pm 1.9$ & $2.8 \pm 2.3$ \\
\hline
\end{tabular}

Values are presented as mean \pm standard deviation.

PSQI, Pittsburg Sleep Quality Index; ESS, Epworth Sleepiness Scale; ISI, Insomnia Severity Scale; BDI, Beck Depression Inventory; MBI, Modified Barthel Index; group 1, good sleeper; group 2, poor sleeper.

a) $\mathrm{p}<0.05$, derived from $\mathrm{t}$-test.

${ }^{b} \mathrm{p}<0.05$, Mann-Whitney $\mathrm{U}$ test between groups 1 and 2 . 
lower function in accordance with the affected side. All scores, except hand grip strength of the dominant hand were significantly different (Table 2).

The average PSQI score in the 80 patients was $8.1 \pm 4.1$, with 57 patients $(71.2 \%)$ having a PSQI score $>5$. The 57 poor sleepers (PSQI scores $>5$ ) had a lower MBI, Purdue peg test, and hand strength test (grip, tip, lateral, and jaw) and a higher 9-hole peg test and BDI scores, compared to the 23 good sleepers.

The average ESS score in the 80 patients was $8.3 \pm 4.4$, with 39 patients $(48.8 \%)$ having an ESS score $>8$. The 39 poor sleepers (ESS scores $>8$ ) had a lower Purdue peg test and hand strength test (grip, tip, lateral, and jaw) and a higher 9-hole peg test and BDI scores, than the 41 good sleepers.

The average ISI score in the 45 patients was $9.0 \pm 7.5$, with 24 patients (53.3\%) having an ISI score $>7$ points. The 24 poor sleepers (ISI scores $>7$ ) had a lower MBI, Purdue peg test, and hand strength test (grip, tip, lateral and jaw) and a higher 9-hole peg test, than the 21 good sleepers. There were no statistically significant differences, with the exception of the BDI scores on the PSQI (Table 3).

PSQI scores were significantly correlated with the BDI scores and grip hand strength and the ESS scores were significantly correlated with the BDI scores. Furthermore, the ISI was significantly correlated with the BDI, MBI, and hand strength test (grip, tip, lateral, and jaw). And the SSS scores were significantly correlated with the BDI, MBI, Purdue peg test, and hand strength test (grip, tip, lateral and jaw) (Table 4).

\section{DISCUSSION}

The observations which we found suggest that mild stroke patients have decreased function of the upper extremity. Poor hand function occurred in the strokeaffected side independent of hand dominance; a finding that was statistically significant. The functional status of mild stroke patients was found to be significantly associated with sleep disturbance and the result presented in the current investigation are consistent with previous studies $[18,21,23]$.

Sleep disturbance can be caused by multiple factors including psychological, personal, and social, and is difficult to assess with simple medical examination because of subjective and individual symptoms [18]. Many clini- cians prefer to assess sleep disturbance such as sleep apnea, insomnia, and daytime sleepiness using systemic medical examinations and questionnaires $[18,29]$.

In the current study, several sleep questionnaires were used (4 in total) that are reflective of different parts. In accordance with results of this study, 3 of the sleep questionnaires indicated that the majority of patients were poor sleepers (PSQI, 71.2\%; ESS, 48.8\%; ISI, 53.3\%). However, it was not possible to measure the sleep state using the SSS as it is primarily used to check the patient's current level of alertness. In 2002, Leppavuori et al. [16] reported that the majority of ischemic stroke patients suffered from insomnia, and Oh and Chun [18] indicated that insomnia was observed in $60.9 \%$ of stroke rehabilitation patients. The current study also showed that patients who were poor sleepers had lower MBI (non-significant), Purdue peg test, 9-hole peg test, and hand strength test (grip, tip, lateral and jaw) compared to those who were good sleepers. Furthermore, sleep status was significantly correlated with functional status. Similarly, Leppavouri et al [16] reported that poor sleeping in ischemic stroke patients was correlated with functional status as measured with the MBI. Oh and Chun [18] reported that their insomnia group had a trend of lower FIM scores compared to their normal group; however, it was not statistically significant. These findings have important implications in stroke patients, because daytime activities and concentration are decreased by sleep disturbance thus resulting in disturbed rehabilitation and delayed functional recovery $[18,21,22]$.

It is possible that handedness could affect upper extremity function and in our study, only three patients were left-handed compared to the 77 patients who were right-handed. A result of this disparity is that it was not possible to compare handedness. However, dominant and non-dominant hand function was reduced in the affected side compared to the healthy side. This means that the patients had a clear functional abnormality and that the functional deterioration of the non-dominant hand was larger than the dominant hand. This may have be explained by the fact that the function of the dominant hand is good in healthy people [39].

The function of the unaffected upper limb also decreased when age was considered and can be explained by disability of hemispheric integration as well as ipsilateral sensory-motor dysfunction [40]. Therefore, evalua- 
tion and treatment of stroke patients with an unaffected side is essential.

Poor sleeping as measured with the PSQI and ESS questionnaires was associated with a higher BDI score compared to those patients who were good sleepers, especially the statistically significant PSQI scores. Goodwin and Devanand [13] referred to the relation between stroke and depression and that these were also associated with functional status. Zavoreo et al. [15] also suggested that motor function and recovery of cognition in stroke patients was adversely affected by depression. In other previous studies, psychological factors such as depression, sleep problems, and fatigue had negative effect on the rehabilitation of stroke patients, which is in similarity with our present results [13-15,18].

Unlike previous studies that assessed functional status using the MBI and FIM, our study focused on assessing the strength and functional status of the upper extremity, especially the hand. While the activities of daily living scale (i.e., the MBI), measures the ability of the patient to perform certain tasks it does not specifically measure the function of the affected limbs [12]. Therefore, this measure is limited to assessing the detailed strength and status of the affected limbs. Upper extremity function is directly associated with activities of daily living in the rehabilitation of stroke patients and is a prognostic factor of functional recovery. This is why the current study focused on assessing the functional status of the upper extremity. Furthermore, mild stroke patients have a large potential to recover their pre-stroke function and for this reason, the assessment of upper extremity function and strength is meaningful for rehabilitation $[9,12]$. Mild stroke patients tend to present with normal looking function with little impairment during the initial periods of rehabilitation. In theory, many mild stroke patients are free from disability [1], in practice, however, many are unable to resume their normal daily life activities. This situation is called hidden dysfunction and therefore, it is important to go for a detailed assessment of the functional status in an effort to remove obstacles and minimize hidden dysfunctions [3]. Edwards et al. [1] suggested that when a stroke patient returns to normal life, psychological and individual factors, such as decreased motivation and depression determine their quality of life and satisfaction. Sleep disturbance is also included in these factors and has a negative effect on quality of life, that is why it is im- portant to perform specific assessments and control the use of medication or sleep hygiene education.

Our study showed that sleep disturbance was correlated with the functional status of the mild acute stroke patients. In a study carried out by Oh and Chun [18], many behavioral and affective disorders appeared at discharge in patients with sleep disturbances and therefore, employment was down. In addition to sleep status, multiple factors such as depression, fatigue, anxiety and environmental factors resulted in decreased activity, concentration, and motivation of the patient and thus recovery of function was delayed $[1,3,14]$. However, our study did not include these factors because only those patients were selected that had no history of depression or mental illness. Further only those patients were taken in which stroke occurred less than 2 weeks from the start of the study, representing acute stroke patients would be less impacted by fatigue. As a result, future studies will be necessary to control for such mood disorders and fatigue.

Sleep disturbance during stroke rehabilitation can be improved by using medication or sleep hygiene education. In the early period of rehabilitation, it is important to assess the patient's sleep cycle and control their sleep condition in order to enhance their functional recovery and social participation. Therefore, future studies have to investigate the effect of sleep control on functional status and recovery in stroke rehabilitation patients. Polysomnography can be used for correct diagnosis, but it is timeconsuming and expensive.

This study included a selected subgroup of stroke patients, which may limit the generalization of the findings. All of the patients had difficulties in completing the different types of sleep questionnaires because many types of sleep questionnaires adversely affected the participation of patients and mild stroke patients relatively did not feel the need to test. Four kinds of questionnaires were used to assess the patient's sleep but the contents of each individual differ and therefore, it was difficult to select a single representative sleep questionnaire. Furthermore, we did not consider environmental factors or pain that may have resulted from the stroke. Future investigations will have to simplify the sleep questionnaires, limit environmental factors, and consider pain.

In conclusion, the affected upper extremity of acute mild stroke patients showed marked deterioration. This study emphasized that sleep disturbance can affect the 
functional status of acute mild stroke patients. Therefore, clinicians must assess the sleep status of acute mild stroke patients and attempt to control it. Further it is important to assist the acute mild stroke patients so that they will smoothly integrate back into society, and restore their pre-stroke condition.

\section{CONFLICT OF INTEREST}

No potential conflict of interest relevant to this article was reported.

\section{ACKNOWLEDGMENTS}

This work was supported by the Soonchunhyang University Research Fund.

\section{REFERENCES}

1. Edwards DF, Hahn M, Baum C, Dromerick AW. The impact of mild stroke on meaningful activity and life satisfaction. J Stroke Cerebrovasc Dis 2006;15:151-7.

2. Green TL, King KM. Functional and psychosocial outcomes 1 year after mild stroke. J Stroke Cerebrovasc Dis 2010;19:10-6.

3. Fowler C. Addressing the work performance of individuals with mild stroke. Am Occup Ther Assoc Spec Interest Sect Q 2013;27:1-4.

4. Carlsson GE, Moller A, Blomstrand C. Consequences of mild stroke in persons $<75$ years: a 1-year followup. Cerebrovasc Dis 2003;16:383-8.

5. Carlsson GE, Moller A, Blomstrand C. A qualitative study of the consequences of 'hidden dysfunctions' one year after a mild stroke in persons $<75$ years. Disabil Rehabil 2004;26:1373-80.

6. Duncan PW, Samsa GP, Weinberger M, Goldstein LB, Bonito A, Witter DM, et al. Health status of individuals with mild stroke. Stroke 1997;28:740-5.

7. Liu-Ambrose T, Pang MY, Eng JJ. Executive function is independently associated with performances of balance and mobility in community-dwelling older adults after mild stroke: implications for falls prevention. Cerebrovasc Dis 2007;23:203-10.

8. Carlsson G. Mild stroke - consequences in everyday life, coping, and life satisfaction [dissertation]. Goteborg, Sweden: the Sahlgrenska Academy at Goteborg
University, Institute of Neuroscience and Physiology; 2007.

9. Au-Yeung SS, Hui-Chan CW. Predicting recovery of dextrous hand function in acute stroke. Disabil Rehabil 2009;31:394-401.

10. Broeks JG, Lankhorst GJ, Rumping K, Prevo AJ. The long-term outcome of arm function after stroke: results of a follow-up study. Disabil Rehabil 1999;21:35764.

11. Parker VM, Wade DT, Langton Hewer R. Loss of arm function after stroke: measurement, frequency, and recovery. Int Rehabil Med 1986;8:69-73.

12. Wade DT, Langton-Hewer R, Wood VA, Skilbeck CE, Ismail HM. The hemiplegic arm after stroke: measurement and recovery. J Neurol Neurosurg Psychiatry 1983;46:521-4.

13. Goodwin RD, Devanand DP. Stroke, depression, and functional health outcomes among adults in the community. J Geriatr Psychiatry Neurol 2008;21:41-6.

14. Park JY, Chun MH, Kang SH, Lee JA, Kim BR, Shin MJ. Functional outcome in poststroke patients with or without fatigue. Am J Phys Med Rehabil 2009;88:554-8.

15. Zavoreo I, Basic-Kes V, Bosnar-Puretic M, Demarin V. Post-stroke depression. Acta Clin Croat 2009;48:32933.

16. Leppavuori A, Pohjasvaara T, Vataja R, Kaste M, Erkinjuntti T. Insomnia in ischemic stroke patients. Cerebrovasc Dis 2002;14:90-7.

17. Mohsenin V, Valor R. Sleep apnea in patients with hemispheric stroke. Arch Phys Med Rehabil 1995; 76:71-6.

18. Oh SY, Chun MH. Insomnia in inpatients during rehabilitation treatment. J Korean Acad Rehabil Med 2000;24:395-401.

19. Brown DL. Sleep disorders and stroke. Semin Neurol 2006;26:117-22.

20. Brooks D, Davis L, Vujovic-Zotovic N, Boulias C, Ismail F, Richardson D, et al. Sleep-disordered breathing in patients enrolled in an inpatient stroke rehabilitation program. Arch Phys Med Rehabil 2010;91:65962.

21. Chon JS, Chun SI, Seo CH, Kim DA, Seok H, Ohn SH. Sleep apnea syndrome and prognosis in stroke patients. J Korean Acad Rehabil Med 2000;24:864-9.

22. Mansukhani MP, Bellolio MF, Kolla BP, Enduri S, Somers VK, Stead LG. Worse outcome after stroke in 
patients with obstructive sleep apnea: an observational cohort study. J Stroke Cerebrovasc Dis 2011;20:401-5.

23. Yan-fang S, Yu-ping W. Sleep-disordered breathing: impact on functional outcome of ischemic stroke patients. Sleep Med 2009;10:717-9.

24. Sohn SI, Kim do H, Lee MY, Cho YW. The reliability and validity of the Korean version of the Pittsburgh Sleep Quality Index. Sleep Breath 2012;16:803-12.

25. Fogelberg DJ, Hoffman JM, Dikmen S, Temkin NR, Bell KR. Association of sleep and co-occurring psychological conditions at 1 year after traumatic brain injury. Arch Phys Med Rehabil 2012;93:1313-8.

26. Cho YW, Lee JH, Son HK, Lee SH, Shin C, Johns MW. The reliability and validity of the Korean version of the Epworth sleepiness scale. Sleep Breath 2011;15:37784.

27. Johns MW. Reliability and factor analysis of the Epworth Sleepiness Scale. Sleep 1992;15:376-81.

28. Bastien $\mathrm{CH}$, Vallieres A, Morin CM. Validation of the Insomnia Severity Index as an outcome measure for insomnia research. Sleep Med 2001;2:297-307.

29. Bailes S, Libman E, Baltzan M, Amsel R, Schondorf $\mathrm{R}$, Fichten CS. Brief and distinct empirical sleepiness and fatigue scales. J Psychosom Res 2006;60:605-13.

30. Quinn TJ, Langhorne P, Stott DJ. Barthel index for stroke trials: development, properties, and application. Stroke 2011;42:1146-51.

31. Sulter G, Steen C, De Keyser J. Use of the Barthel index and modified Rankin scale in acute stroke trials. Stroke 1999;30:1538-41.

32. Viinamaki H, Tanskanen A, Honkalampi K, Koivu-
maa-Honkanen $\mathrm{H}$, Haatainen $\mathrm{K}$, Kaustio O, et al. Is the Beck Depression Inventory suitable for screening major depression in different phases of the disease? Nord J Psychiatry 2004;58:49-53.

33. Kamimura T, Ikuta Y. Evaluation of sustained grip strength for a stroke patient with a mild paresis. Hiroshima J Med Sci 2002;51:23-31.

34. Kamimura T, Ikuta Y. Evaluation of grip strength with a sustained maximal isometric contraction for 6 and 10 seconds. J Rehabil Med 2001;33:225-9.

35. Hammer A, Lindmark B. Test-retest intra-rater reliability of grip force in patients with stroke. J Rehabil Med 2003;35:189-94.

36. Gallus J, Mathiowetz V. Test-retest reliability of the Purdue pegboard for persons with multiple sclerosis. Am J Occup Ther 2003;57:108-11.

37. Schaechter JD, Perdue KL. Enhanced cortical activation in the contralesional hemisphere of chronic stroke patients in response to motor skill challenge. Cereb Cortex 2008;18:638-47.

38. Lin KC, Chuang LL, Wu CY, Hsieh YW, Chang WY. Responsiveness and validity of three dexterous function measures in stroke rehabilitation. J Rehabil Res Dev 2010;47:563-71.

39. Kim TY, Kang SY, Kim HS, Shin BS. Hand strength and dexterity evaluation with age. J Korean Acad Rehabil Med 1994;18:780-8.

40. Kim HW, Ko YJ, Kang SY, Suh SS. Effects of unilateral brain damage on ipsilateral upper extremity function in hemiplegia. J Korean Acad Rehabil Med 2000;24:813. 